\title{
Dad's diet lives on
}

Two recent studies in rodents show that unhealthy paternal diets can reprogramme gene expression in offspring, implicating epigenetics in these transgenerational effects.

$\mathrm{Ng}$ and colleagues fed male rats a high-fat diet and looked for effects in their adult female offspring, which were fed a normal diet. These daughters had normal body fat but showed signs of pancreatic $\beta$-cell impairment and altered expression (as compared to controls) of 642 genes that are involved in pathways related to insulin regulation and glucose metabolism. The gene with the greatest alteration in expression was interleukin-13 receptor- $\alpha 2$ (Il13ra2), which is implicated in regulating pancreatic cell function. Interestingly, DNA methylation at a cytosine residue close to the ll13ra2 transcriptional start site was reduced in these females.

Carone and colleagues looked at the effect of a paternal low-protein diet in mice. Offspring of both sexes showed altered gene expression compared to controls, including genes involved in fat and cholesterol biosynthesis, consistent with physiological differences in these mice. Modest changes in DNA methylation were seen at many sites, including a reproducible change close to the Ppara gene, which encodes peroxisome proliferator-activated receptor- $\alpha$, a regulator of lipid metabolism.

Although there is increasing evidence for effects of parental environment in offspring, these studies add to just a handful of cases in which the molecular basis has been at least partly elucidated. Clearly, the role of epigenetics in such transgenerational effects will be an important focus of future studies.

Louisa Flintoft

ORIGINAL RESEARCH PAPERS Ng, S.-F. et al

Chronic high-fat diet in fathers programs $\beta$-cell dysfunction in female rat offspring. Nature 467 963-966 (2010) | Carone, B. R. et al. Paternally induced transgenerational environmental reprogramming of metabolic gene expression in mammals. Cell 143, 1084-1096 (2010) 